\title{
ERRATUM
}

\section{Erratum to: Repeat Abortions in New York City, 2010}

\author{
Amita Toprani, Betsy L. Cadwell, Wenhui Li, Judith Sackoff,
} Carolyn Greene, and Elizabeth Begier

\section{Erratum to: J Urban Health \\ DOI 10.1007/s11524-015-9946-3}

Please note that the authors for this article are:

Amita Toprani ${ }^{1,2}$, Betsy L. Cadwell ${ }^{3}$, Wenhui $\mathrm{Li}^{1}$, Judith Sackoff ${ }^{1}$, Carolyn Greene ${ }^{1}$, and Elizabeth Begier ${ }^{1}$.

Author affiliations:

1. New York City Department of Health and Mental Hygiene, New York, NY, USA

2. Epidemic Intelligence Service, Centers for Disease Control and Prevention, Atlanta, GA, USA

3. Scientific Education and Professional Development Program Office, Centers for Disease Control and Prevention, Atlanta, GA, USA

The online version of the original article can be found at http://dx.doi.org/10.1007/s11524-015-9946-3.

Toprani, Li, Sackoff, Greene, and Begier are with the New York City Department of Health and Mental Hygiene, New York, NY, USA; Toprani is with the Epidemic Intelligence Service, Centers for Disease Control and Prevention, Atlanta, GA, USA; Cadwell is with the Scientific Education and Professional Development Program Office, Centers for Disease Control and Prevention, Atlanta, GA, USA.

Correspondence: Amita Toprani, New York City Department of Health and Mental Hygiene, New York, NY, USA. (E-mail: atoprani@health.nyc.gov) 